\title{
Criminologie
}

\section{La sécurité privée et le problème de la confiance : l'expérience australienne}

\section{Tim Prenzler}

Volume 31, numéro 2, automne 1998

La sécurité privée

URI : https://id.erudit.org/iderudit/017420ar

DOI : https://doi.org/10.7202/017420ar

Aller au sommaire du numéro

Éditeur(s)

Les Presses de l'Université de Montréal

ISSN

0316-0041 (imprimé)

1492-1367 (numérique)

Découvrir la revue

Citer cet article

Prenzler, T. (1998). La sécurité privée et le problème de la confiance :

l'expérience australienne. Criminologie, 31(2), 87-109.

https://doi.org/10.7202/017420ar
Résumé de l'article

The private security industry in Australia, as in many jurisdictions, has been beset by allegations of fraud, abuse of powers and incompetence. These problems are illustrated through trade practices, prosecutions, government inquiries, incident reports and other sources. Causation is analysed in terms of Shapiro's (1987) formulation of the problem of "policing trust". The substantial delegation of responsibility entailed in security work makes it highly vulnerable to exploitation and fraud, and this potential is exacerbated by the inadequate regulation of security services. Mitigation of these problems is suggested though an enhanced partnership approach of government and the security industry to regulatory control, an through an assessment of the benefits of in-house security. 


\section{LA SÉCURITÉ PRIVÉE ET LE PROBLÈME DE LA CONFIANCE : L'EXPÉRIENCE AUSTRALIENNE Tim Prenzler}

The private security industry in Australia, as in many jurisdictions, has been beset by allegations of fraud, abuse of powers and incompetence. These problems are illustrated through trade practices, prosecutions, government inquiries, incident reports and other sources. Causation is analysed in terms of Shapiro's (1987) formulation of the problem of "policing trust". The substantial delegation of responsibility entailed in security work makes it highly vulnerable to exploitation and fraud, and this potential is exacerbated by the inadequate regulation of security services. Mitigation of these problems is suggested though an enhanced partnership approach of government and the security industry to regulatory control, an through an assessment of the benefits of inhouse security.

\section{INTRODUCTION}

En Australie, la sécurité privée est une industrie importante et en pleine expansion. Elle fournit des services essentiels de protection des biens contre le vol et les dommages et de protection des personnes contre les agressions. Cependant, la sécurité, par définition, repose sur des prémisses de confiance et des prises de responsabilités. C'est, précisément, la nature de la relation entre ces deux termes qui crée des occasions d'abus de confiance.

L'industrie australienne de la sécurité privée présente une piètre image en raison des allégations périodiques de fraude, d'infiltration par des criminels et de prestations de service de mauvaise qualité formulées à son endroit. Bien qu'il soit difficile de prouver bon nombre de ces allégations, il existe suffisamment de sources d'information dans les archives publiques, tels les rapports d'enquête et d'importants récits de fraudes, pour faire la démonstration d'un haut risque d'inconduite et de dissimulation.

Compte tenu du risque élevé d'abus, il est surprenant de constater que la sécurité privée est l'une des industries les moins réglementées en Australie.

1. School of Justice Administration, Griffith University, 4111, Brisbane, Australie. 
À l'intérieur comme à l'extérieur de l'industrie, un large consensus s'est en effet établi quant à l'inefficacité des contrôles actuels ; on reconnaît que la réglementation est partielle, fragmentaire et incohérente d'une région à l'autre, en plus d'être minime. Une approche globale du problème, à l'échelle nationale, paraît nécessaire pour rehausser les normes actuelles.

\section{L'ÉTUDE}

L'étude dont il sera question ici s'est justement donné pour objet de cerner les principaux types de problèmes rencontrés dans le domaine de la sécurité, en particulier ceux posés par les contrats de services de sécurité, et d'élaborer les principes d'un cadre de réglementation visant à minimiser ces problèmes. Son contexte est celui 1) de l'expansion des services de sécurité ; 2) de l'évolution de la législation sur la sécurité en Australie vers un plus grand contrôle ; 3 ) des allégations répétées de délits et d'inconduites dans l'industrie, principalement dans les domaines qui échappent presque entièrement à toute forme de contrôle.

\section{Les sources de données}

Les sources de données utilisées aux fins de notre étude sont essentiellement qualitatives. Elles comprennent des enquêtes judiciaires, des études menées par la police et les organismes de réglementation, une cause civile, et des récits d'enquêtes journalistiques. Ces données sont représentatives des divers types de problèmes rencontrés dans les différentes régions du pays. Les rapports consultés font tous partie du domaine public et sont disponibles sur demande. Il est par contre plus difficile d'obtenir des preuves tangibles de types d'abus généralisés. Ceci résulte sans doute de la nature même de la relation entre les prestataires de services de sécurité privée et leurs clients, ce qui laisse à penser que les cas présentés illustrent peut-être un problème plus profond d'inconduite systémique ${ }^{2}$.

\section{LES PROBLÈMES DES SERVICES DE SÉCURITÉ}

\section{L'industrie de la sécurité en Australie}

Dans une revue internationale de la littérature, George et Button (1997) font référence au caractère quasi universel des tendances de l'industrie de la sécurité. L'Australie semble avoir suivi le modèle répandu de croissance fulgurante entre les années 1960 et la fin des années 1980. Par exemple, dans l'État de Victoria, entre 1980 et 1990 , le nombre d'entreprises et d'agents

2. Pour une étude similaire menée en Angleterre, voir South, 1983. 
de sécurité possédant un permis a presque doublé et le nombre d'enquêteurs a triplé (Golsby, 1998). La croissance a ralenti, mais a néanmoins atteint un point où les agents de sécurité sont au moins deux fois plus nombreux que les policiers conventionnels.

L'identification d'un profil de l'industrie australienne est rendue difficile en raison du problème courant du manque de fiabilité des statistiques officielles, et aussi parce que les fonctions des agents de sécurité sont réparties en diverses catégories professionnelles et selon des systèmes de permis différents d'un État à l'autre. Les données les plus récentes émanant du Australian Bureau of Statistics (ABS, 1996) indiquent que l'on trouve en Australie 30556 gardiens et agents de sécurité - soit environ les deux tiers du nombre de policiers d'État et fédéraux. Ce chiffre peut sembler modeste pour un pays qui a une population de 18 millions d'habitants. Cependant, selon un rapport sur l'industrie de la sécurité, la Nouvelle-Galles du Sud (le plus grand État de l'Australie avec une population de six millions d'habitants) comptait 37354 personnes détenant un permis de sécurité en 1994, soit plus de deux fois le nombre d'agents de police (NSWPS, $1995: 3$ ). Malgré la confusion qui règne en matière de chiffres, on peut affirmer que le personnel des services de sécurité est devenu presque omniprésent, en Australie comme dans nombre d'autres pays (Stenning, 1994).

L'industrie de la sécurité présente l'image d'un secteur composé d'un grand nombre de petites compagnies éphémères. Le ABS Business Register de 1995 enregistrait 1633 Services de sécurité et d'enquêtes qui employaient moins de 10 personnes, et 37 compagnies qui comptaient 100 employés et plus. Le nombre total de compagnies était de 2010 (ABS, 1997).

Cependant, l'industrie australienne a été dominée par quelques grandes entreprises (Johnston, 1992). Les seules données détaillées dont nous disposons, tirées d'une étude spéciale menée par ABS en 1988, ont révélé que $1 \%$ des entreprises de sécurité avaient le contrôle de $66 \%$ des emplois et étaient responsables de $60 \%$ des changements de personnel en matière de sécurité privée (ABS, 1988). La tendance à l'oligopole s'est accéléré au cours des années 1990 avec l'achat, par Tempo, du Groupe Securitas et la disparition de Wormald, Mayne Nickless, James Hardie et MSS du marché suite à des fusions opérées par la compagnie Chubb (Courier Mail, 5 décembre 1995 ; Security Australia, juin 1996). L'Australian Competition and Consumer Commission (ACCC) a accepté ces fusions, déclarant qu'il y avait peu d'obstacles pour les nouveaux venus en raison des faibles investissements nécessaires à la mise sur pied d'une entreprise de services de sécurité (ACCC, 1996b : 21-22). L'ACCC ne s'est pas rendu compte du caractère ironique de cette affirmation, dès lors qu'il était question de contrôle de la qualité. L'achat par Chubb de la branche des services de construction de 
James Hardie a été décrit par Security Australia comme illustrant " toute l'étendue de la détermination du groupe Chubb de dominer complètement les marchés australiens des services de sécurité et d'incendie » (janvier 1997).

\section{Problèmes et questions}

La croissance et l'expansion de l'industrie de la sécurité soulèvent des questions complexes sur les implications sociales et légales de l'accroissement du contrôle social par des agences non policières. Shearing et Stenning (1983), de même que Swanton (1993), soutiennent que l'accroissement de la privatisation de la sécurité entraînera l'apparition de deux systèmes de justice : un meilleur service pour ceux qui ont les moyens de payer et un pauvre cousin subventionné par les fonds publics pour ceux qui ne sont pas en mesure de le faire. En outre, un pourcentage croissant de crimes et de désordres publics sera traité par les services privés plutôt que par le système de justice pénale. On pourrait dire qu'une sécurité axée sur la non-application de la loi est justifiable puisqu'elle évite des poursuites onéreuses au résultat incertain, et met l'accent sur la prévention plutôt que sur la rétribution ultérieure. C'est cependant-là un scénario optimiste. Le manque de gestion, de formation et de contrôle adéquats pourrait se traduire par l'application d'une justice sauvage (vigilante justice) par les agents de sécurité, sans les garanties offertes par le droit ou par des stratégies de prévention responsables. L'industrie de la sécurité privée, qui entretient des relations de courte durée avec ses clients, pourrait être facilement portée à ignorer des éléments comme les garanties procédurales et le respect des libertés individuelles (Sarre, 1994 ; Reynolds et Winson, 1996).

Les allégations de malhonnêteté adressées à l'industrie de la sécurité en Australie sont fréquentes. Dans un récent débat écrit sur la réglementation de l'industrie, présenté dans le Australian Capital Territory, on décrivait les " problèmes graves " causés par la croissance de ce secteur et on notait que « la violence et les allégations d'infiltration criminelle ont nui à l'industrie » (ACT, $1992: \mathrm{i}$ ). On qualifie souvent de vauriens, de voyous et de criminels certains éléments de l'industrie (par exemple, Wilson, 1994 ; Rent-a-Cop, 1995). En 1994, le Victorian Deputy Registrar for Private Agents affirmait que les entreprises de sécurité « ont des méthodes de sélection médiocres. Elles ne possèdent aucun système hiérarchique de supervision. Les employés sont envoyés sur le terrain pour faire le travail et on ne les revoit plus avant la fin de leur travail. Les normes de formation sont tout à fait insatisfaisantes » (Private Cops, 1994). Des préoccupations du même ordre ont été exprimées, aussi bien à l'intérieur qu'à l'extérieur de l'industrie (Rees, 1984 ; Morley, 1994 ; Prenzler, 1995). La partie qui suit traite de ces problèmes plus en détail. 


\section{Études de cas d'inconduites}

\section{La fraude et l'incompétence des patrouilles et des services d'alarme}

Au milieu des années 1990, dans le Newcastle, les employés de Chubb dénoncent une fraude comportant la falsification des données de vérification de patrouilles de sécurité sur les lieux surveillés, dont un bâtiment du gouvernement. La fraude dure, semble-t-il, depuis au moins dix ans. Un informateur affime avoir transmis cette information à un haut fonctionnaire de l'État, et ajoute qu'aucune mesure n'a été prise. Bien que le haut fonctionnaire soutienne avoir réglé le problème - par le renvoi du cadre responsable - certains employés déclarent que les ressources sont toujours insuffisantes pour superviser correctement les vérifications de sécurité.

De même, le Weekend Independent de Brisbane signale : «Si vous engagez une entreprise de sécurité pour patrouiller vos locaux commerciaux durant la nuit, il y a de fortes chances que l'on vous leurre ». Les journalistes d'enquête révèlent alors que dix entreprises de sécurité fournissent seulement une partie des services contractés pour des vérifications de sécurité de nuit. Ces révélations donnent lieu à une poursuite en cour fédérale par la Trade Practices Commission contre Mayne Nickless, à l'époque une des plus grandes entreprises de sécurité de l'Australie, pour fraude et déclarations mensongères. Plusieurs de ces sociétés étaient membres de l'Australian Security Industry Association Limited (ASIAL).

À Perth, la ACCC découvre que Wormald (maintenant négoce de Chubb) omet systématiquement d'effectuer les vérifications de patrouilles mobiles selon les ententes convenues avec les clients. En 1996, Chubb est forcé d'accepter les engagements exécutoires de la Cour l'obligeant à se doter de personnel et de personnes-ressources en nombre suffisant pour assurer un service adéquat à ses clients (Sunday Mail, 18 septembre 1994 ; Weekend Independent, 30 septembre 1994 ; TPC, 1995).

Dans le Queensland et le nord de la Nouvelle-Galles du Sud, la Trade Practices Commission a enquêté sur l'opération d'un système d'alarme contre les cambrioleurs et d'urgence 24 heures - le Neva Alone - destiné à protéger les personnes âgées et handicapées et sous la reponsabilité du MSS, alors filiale de Mayne Nickless. L'enquête a montré qu'il arrivait fréquemment que les signaux d'alarme ne sonnent pas au poste de contrôle, et que le personnel était parfois absent. Le poste de contrôle recevait des rapports de quelque 4000 points de contact mais utilisait des systèmes informatiques incompatibles. En outre, il arrivait souvent que le système soit en panne. Des entreprises et des particuliers ont logé de nombreuses plaintes et la compagnie Telecom a été blâmée. Le personnel changeait continuellement et les informateurs ont déclaré que les cadres intermédiaires et supérieurs 
étaient hostiles à l'égard des employés qui portaient plainte (Rent-a-Cop, 1995 ; ACCC, 1996a).

En 1997, une étude menée dans l'ouest de l'Australie sur l'efficacité des dispositifs d'alarme antivol a révélé que la police avait répondu à 45000 appels pour déclenchement d'alarme en 12 mois. Sur ce nombre, $94 \%$ des demandes n'auraient pas nécessité une intervention policière. On a estimé que 900 appels par semaine étaient dus à des erreurs commises par les usagers, des équipements défectueux, des installations incorrectes, un manque d'entretien ou certaines conditions climatiques. Ce gaspillage massif des fonds publics a nécessité la formation d'une unité de police de la sécurité, de la propre initiative de la police, pour établir un programme de répartition des interventions entre la police et les compagnies de sécurité responsables (Security Australia, février 1997 ; WAP, 1997).

\section{Employés sous-payés}

Depuis de nombreuses années, on allègue que l'industrie des services de sécurité australienne sous-paie ses employés. À ce sujet, un directeur d'une compagnie de sécurité ayant son siège social à Sydney a demandé la création d'une association des entreprises qui assurent le paiement du salaire minimum. Le directeur a soutenu que les « ministères, pressés de réduire leurs dépenses, figurent parmi les pires délinquants en acceptant régulièrement des soumissions d'entreprises tellement basses que l'on peut-être certain d'emblée que les employés ne recevront pas le salaire minimum " (Security Australia, mars 1993 : 25). Les préoccupations à ce sujet ont incité le ministère de l'Éducation de la Nouvelle-Galles du Sud à préciser dans ses contrats avec les entreprises de sécurité que ces dernières devaient verser au moins le salaire minimum à leurs employés (Rent-a-Cop, 1995). Une enquête menée par la police de la Nouvelle-Galles du Sud sur l'industrie de la sécurité a révélé « plusieurs cas de malversation en matière d'exploitation du personnel de sécurité » (NSWPS, 1995 : 9). Il s'est toutefois avéré impossible d'engager des procédures de révocation du permis contre ies délinquants. Le problème concernait des déclarations mensongères quant à la rémunération — inférieure au salaire minimum - indiquée pour les coûts de main-d'œuvre dans les contrats avec les clients et l'utilisation abusive du système de subvention Job Start, se concluant par le renvoi des employés à la fin de la période de subvention. Cette dernière pratique a été sévèrement condamnée dans le rapport :

La procédure... produit un certain nombre d'effets négatifs sur l'industrie et le personnel de sécurité. En premier lieu, elle décharge dans la collectivité des centaines, si ce n'est des milliers d'agents de sécurité possédant un permis dans un marché offrant peu de perspectives 
d'emploi. Elle détruit les rêves, les ambitions et les aspirations de personnes qui espèrent faire carrière dans l'industrie de la sécurité. Elle crée un marché injuste de soumission de contrats de sécurité. Enfin, bien que cela puisse améliorer artificiellement les statistiques de chômage du Commonwealth Employment Service, cette attitude est essentiellement malhonnête (NSWPS, $1995: 10$ ).

Un sondage récent mené auprès des gestionnaires de la sécurité dans le Queensland a montré un appui solide en faveur de la surveillance du paiement du salaire minimum - ce qui indique une réelle préoccupation quant à la rémunération inférieure au salaire minimum et aux pratiques concurrentielles de sous-évaluation des soumissions (Prenzler, Hayes et Wortley, en préparation).

\section{Accès illégal aux renseignements personnels}

La première étude sur les membres de l'industrie de la sécurité en Australie (Rees, 1984) a révélé que les agents de sécurité privée, en particulier lorsqu'il s'agissait d'ex-policiers, avaient facilement accès à de l'information confidentielle. Cette pratique a été confirmée dans le rapport de 1992 de la NSW Independent Commission Against Corruption (ICAC), qui a déclaré que les agents d'investigation privés servaient d'intermédiaires dans un «commerce illicite massif » de renseignements confidentiels qui excédait probablement un million de dollars sur un certain nombre d'années. On a identifié plus de 250 personnes impliquées, y compris des policiers, des fonctionnaires de plusieurs ministères (Sécurité sociale, Impôt, Enregistrement des véhicules, Telecom, Postes et Medicare), ainsi que des banques, des compagnies d'assurances et autres établissements financiers et des enquêteurs privés. L'information servait à préparer les causes civiles, à dénicher les débiteurs et «à d'autres fins plus sinistres ». La Commission a reconnu un élément de « juste cause » dans ces enquêtes, mais a aussi constaté que de graves violations de la loi s'étaient produites et que la prolifération des moyens illégaux d'accès aux renseignements contrecarraient les pressions pour la réforme du droit. Les intrusions touchaient divers domaines de la vie privée, par exemple, des questions sur la nature des avantages reçus, des détails sur les passeports et comptes bancaires, sur la correspondance, ainsi que la divulgation de numéros de téléphone non publiés. Souvent, les personnes dont les dossiers étaient fouillés n'étaient pas les cibles directes des enquêtes. Par ailleurs, certains destinataires de l'information étaient des criminels connus et, dans de nombreux cas, les agents ne savaient pas à quoi serviraient les renseignements transmis.

Le cas suivant illustre les conséquences involontaires possibles des activités des enquêteurs privés. Cet exemple est tiré des archives des années 
1985 et 1986 de l'Office du coroner de Victoria. Une femme, R.B., est la conjointe de fait d'un homme, G.K. La relation devient conflictuelle : G.K. se montre très possessif et violent et, à la suite d'un appel de R.B. à la police, est accusé de voie de fait. Craignant pour sa vie, R.B. s'enfuit de la maison. Comme elle craint que G.K. ne la retrouve et tente de la tuer, elle demande au déménageur de ne pas divulguer son adresse. Ses tentatives de retrouver R.B. ayant échoué, G.K. engage un détective privé qui réussit à localiser R.B. et en informe son client. G.K. se présente au nouveau domicile de R.B. et la tue de plusieurs coups de feu. Il quitte l'Australie et ne sera jamais traduit en justice (Polk et Ranson, 1991).

\section{Violence dans les débits de boisson}

À Victoria, à la fin des années 1980, les préoccupations concernant l'escalade de la violence dans les boîtes de nuit et les hôtels ont incité la tenue d'une enquête. Le rapport du Victorian Community Council Against Violence (VCCAV, 1990) indique la présence de voies de fait graves, de bagarres et de harcèlement dans les débits de boisson et lieux avoisinants. On estime que $20 \%$ de toutes les voies de fait graves rapportées dans l'État - soit environ 818 par année - sont associées aux débits de boisson. Un sondage auprès des clients des boîtes de nuit révèle que " la vaste majorité " d'entre eux ont été témoins d'actes de violence ; $30 \%$ soutiennent en avoir été victimes et, parmi eux, $37 \%$ déclarent que les actes de violence étaient commis par le personnel de sécurité (les contrôleurs de foule ou les videurs). Une partie de la narration du responsable adjoint des agents privés est particulièrement imagée :

En août 1990, dans un grand hôtel de banlieue, 24 voies de fait graves ont été commises par des videurs contre des clients dans une seule période de 28 jours. Dans chaque cas, les blessures infligées par les videurs ont nécessité une hospitalisation. Dans la majorité des cas, il s'agissait d'attaques soutenues de plusieurs videurs contre un seul client. De nombreuses scènes, enregistrées sur bande vidéo, montrent les videurs martelant à coups de poing, coups de pied et à coups de tête des querelleurs déjà à demi-inconscients, avant de les traîner à l'extérieur pour les jeter sur la rue ou sur le trottoir. Il est fréquent que les victimes soient par la suite agressées à l'extérieur des lieux et occasionnellement poursuivies sur une certaine distance, les videurs continuant à les battre (NSWPS, 1995 : 11).

L'enquête a montré que la déréglementation des débits de boisson, en 1987, avait contribué à la prolifération de ces établissements mal gérés, auxquels on permettait de rester ouverts toute la nuit, et de servir des boissons bon marché. On a établi un lien étroit entre la violence et la surconsommation 
d'alcool, résultant de pratiques de gestion irresponsables, comme certaines méthodes encourageant à boire, les boissons offertes aux clients ivres, l'entassement des clients et les spectacles de mauvais goût. On a également reproché à la direction d'encourager ou de tolérer que les préposés à la sécurité aient recours à l'agression, à une certaine forme de justice sauvage et à la supervision des bagarres. En outre, l'ivresse et l'agressivité des clients amèneraient également ceux-ci à s'attaquer aux préposés à la sécurité. L'enquête a aussi établi que, dans Melbourne, certaines nuits, on comptait entre quatre et dix agents de sécurité pour chaque tranche de 1000 clients. Or, il n'existait ni formation obligatoire ni normes établies de formation pour ces agents. Il était par conséquent pour ainsi dire impossible de tenir quiconque légalement responsable de quoi que ce soit en raison de la nature temporaire de l'emploi, de la mobilité du personnel, des difficultés à établir l'identité des fautifs, de l'ivresse des clients et des témoins, de l'indifférence et de la peur de représailles.

\section{L'échec des solutions d'ordre légal}

Depuis quelques années, les compagnies d'assurances australiennes ont tendance à demander un dédommagement aux entreprises de sécurité négligentes à la suite de la perte de biens après un cambriolage ou un autre délit. La plupart de ces poursuites se règlent à l'amiable pour éviter au fournisseur de services de sécurité d'avoir à comparaître en Cour, ce qui créerait peutêtre un précédent (Sarre, 1998). Parmi les quelques cas jugés en Cour, la majorité se règlent en faveur du plaignant, mais pas toujours de façon satisfaisante. Une de ces causes, engagée à Brisbane à la suite d'un cambriolage, a donné lieu à une poursuite intentée conjointement par l'assureur et l'assuré contre l'installateur du dispositif d'alarme, la compagnie de surveillance du signal d'alarme et la compagnie chargée de la patrouille de sécurité. Le magistrat a donné raison au plaignant. Cependant, entre le moment où le cambriolage a eu lieu (août 1992) et la fin des audiences (octobre 1994), l'assureur et l'installateur du système d'alarme avaient fait faillite (Lumley General Insurance Ltd, M. Marsh et A. K. Bushnell contre G. Rosenberg and Ors, 1994).

\section{La formation des criminels}

Le rapport de la police de la Nouvelle Galles du Sud sur l'industrie de la sécurité privée comprend une section sur la formation des installateurs de systèmes d'alarme électronique intitulée : «La formation des criminels ». L'étude a révélé que des personnes possédant un casier judiciaire pour cambriolages et vols à main armée pouvaient suivre une formation et obtenir un permis d'installation de systèmes d'alarme. La formation comprenait les 
moyens de contourner et de supprimer les dispositifs de sécurité (NSWPS, 1995 : 15). Le rapport fait également état d'une cause où, s'étant vu refuser l'octroi d'un permis de garde de sécurité par le Bureau d'enregistrement des permis, un contrevenant détenu pour vol à main armée, en détention pendant les week-ends, s'est adressé à la cour d'appel... qui lui a octroyé son permis (Rent-a-Cop, 1995).

\section{Sous le feu des belligérants}

La question de l'octroi des permis de port d'armes et de la formation nécessaire pour s'en servir a fait l'objet d'une attention soutenue. Dans une enquête menée par la police de la Nouvelle-Galles du Sud, on cite des instructeurs de cours d'armes à feu qui estiment que le cours d'une journée imposé aux gardes de sécurité est « totalement insuffisant », tant en ce qui concerne les habiletés pratiques que la connaissance des dispositifs légaux régissant la possession et l'utilisation d'armes à feu. Les instructeurs déclarent qu'il « est téméraire de mettre un pistolet entre les mains d'une personne si mal entraînée » (NSWPS, 1995 : 23) et décrivent l'attitude de certaines compagnies distribuant inconsidérément des armes à feu comme étant « pour le moins désinvolte ». En outre, il semble que certains agents de sécurité sous le coup d'une ordonnance pour violence appréhendée circulent avec des armes à feu de fonction. En 1995, le Bureau des permis d'armes à feu de la Nouvelle-Galles du Sud indiquait qu'au cours d'une période de 12 mois, il avait examiné sept cours de formation et en avait annulé cinq en raison de pratiques inadéquates (Rent-a-Cop, 1995).

Dans le Queensland, en 1995, trois jours avant Noël, Brenda Scott, âgée de 27 ans, se rendait à la banque, située dans une rue commerçante de la banlieue Moorooka de Brisbane, lorsque deux hommes armés ont tendu une embuscade et attaqué une voiture blindée. Un des hommes a par la suite prétendu qu'un agent de sécurité avait tiré sur lui avant que l'échange de coups de feu ne commence entre les deux groupes. Un homme de 71 ans a été atteint d'une balle au bras, un touriste a reçu une décharge dans la jambe, un garde de sécurité a été blessé au doigt et Brenda Scott a reçu un coup de feu dans le dos. Un témoin a décrit ainsi la fusillade : "des coups de feu, suivis de coups de feu et encore de coups de feu... C'était comme un film de cowboys, il y avait des coups de feu partout ». La balle qui a atteint Brenda Scoot s'est logée dans la colonne vertébrale, provoquant une paralysie permanente. Par la suite, il a été impossible d'établir si le coup de feu provenait d'un des hommes armés ou d'un agent de sécurité. À la suite de ce drame, le ministre de la Sécurité a critiqué les façons cow-boy de l'industrie et a exigé des contrôles plus rigoureux (Courier Mail, 23 décembre 1995, 24 novembre 1997, 3 décembre1997, 7 décembre 1997). 
Lors d'un incident similaire, en 1987, à Adélaïde en Australie méridionale, une personne qui faisait ses courses dans une rue achalandée a été atteinte d'une balle tirée par un agent de sécurité en direction de cambrioleurs. Le garde a été accusé en cour criminelle. Le magistrat qui entendait la cause a décrit l'intervention du garde comme «criminellement stupide», mais a rendu une ordonnance de non-lieu parce que les preuves étaient insuffisantes pour maintenir l'accusation de coups et blessures (Sarre, 1998).

Ces incidents, de même que plusieurs autres fusillades impliquant des gardes de sécurité, ont poussé le gouvernement à enquêter sur l'industrie du transport de l'argent. Un rapport boiteux a été produit en décembre 1996 sans aucune référence directe au cas de Moorooka. Le secteur des voitures blindées de l'industrie a mis l'accent sur la vulnérabilité des agents qui doivent franchir à pied, avec des objets de valeur, la distance qui sépare la voiture blindée de l'établissement. L'idée d'imposer certains aménagements urbains afin de pallier le problème a été considérée par la commission d'enquête, mais elle a finalement été mise de côté parce qu'elle ne convenait pas aux établissements financiers, aux commerces et aux constructeurs de centres commerciaux (QIRC, 1996). Dans le même ordre d'idée, un rapport de la Commission industrielle de la Nouvelle Galles du Sud concernant l'industrie du transport d'argent, qui critiquait largement la négligence à l'endroit de la sécurité du public et l'absence de planification architecturale pour la remise des objets de valeur, ne recommande rien de plus que des évaluations des risques encourus dans les lieux de livraison (IRCNSW, 1996).

\section{ANALYSES}

\section{Le problème de la confiance}

Les données rapportées ci-dessus relatent des incidents isolés ou des études se limitant à une unité administrative particulière. On pourrait soutenir que ces cas ne sont pas représentatifs de l'industrie de la sécurité dans son ensemble, ou qu'ils ne concernent pas uniquement la sécurité. Les problèmes de fraude, de négligence et d'incompétence sont courants dans toutes les sphères d'activités commerciales. Rien ne justifierait donc l'adoption de lois spéciales pour l'industrie de la sécurité. Il s'agirait simplement d'une question de surveillance des consommateurs et de vigilance accrue des autorités en place. Par ailleurs, on pourrait prétendre que ces cas, comme d'autres qui ne sont pas présentés ici, ne constituent que la pointe de l'iceberg. L'Australie n'a pas mené une enquête nationale auprès de cette industrie avec tous les pouvoirs consentis à une commission royale d'enquête. Les enquêtes et les études entreprises ont cerné une grande variété de problèmes, dont certains très répandus, et on peut craindre que ce qui se produit dans un État risque 
fort de se produire ailleurs. En outre, la critique la plus tranchante envers l'industrie est l'industrie elle-même. Ses membres ont exprimé à maintes reprises des préoccupations, à titre d'informateurs privilégiés, au sujet de la conduite des autres membres (Prenzler, 1995 ; Prenzler, Baxter et Draper, 1997).

Ce que les études illustrent surtout, c'est sans doute le potentiel d'abus dont les clients peuvent être victimes ainsi que leur vulnérabilité. Il existe de nombreuses compagnies de sécurité hautement professionnelles et de bonne réputation qui assurent un excellent service à leurs clients. Le problème du consommateur est de choisir une entreprise fiable et de surveiller la qualité du service. Voilà le défi que doivent relever les compagnies et les personnes qui font appel à des entreprises de sécurité. Mais il y a également une tierce partie, beaucoup plus vaste et directement concernée : le personnel des entreprises de sécurité, les personnes qui font leurs courses et qui sont exposées aux fusillades entre gardes de sécurité et bandits, les clients qui désirent passer une soirée de spectacle agréable, les personnes qui font l'objet d'enquêtes de la part d'agents de sécurité privée et dont les renseignements personnels sont conservés dans des banques de données des agences gouvernementales, les victimes de violence conjugale qui se cachent et les personnes qui font des appels d'urgence et sont forcées d'attendre la police occupée ailleurs à régler des cas de fausses alarmes.

Pour tous ces groupes, les services de sécurité symbolisent le problème de la surveillance des relations de confiance, selon la formulation de Shapiro (1987). Les entrepreneurs du contrôle social privé à gages présentent des problèmes particuliers au chapitre de la délégation de la responsabilité de la protection et du partage des secrets confiés par des personnes vulnérables :

Le réseau des relations créé par les agences est structurellement précaire et susceptible d'abus car les agents ont la garde d'un bien qui ne leur appartient pas, un pouvoir discrétionnaire quant aux interventions et la possibilité de créer et de diffuser de l'information que les destinataires ne peuvent vérifier, faute d'accès ou de savoir-faire. Qui plus est, les rôles des agents comportent des tentations inhérentes à leur fonction ; on ne sait jamais si les agents vont choisir d'agir dans l'intérêt de leurs clients (ou de leurs commettants, dans le jargon juridique) ou profiter, pour leur propre avantage, du pouvoir, de leur capacité d'accès à l'information et des riches dont ils ont la confiance (Shapiro, $1987: 200$ ).

Le problème est exacerbé par une complexité technologique et bureaucratique accrue qui dépersonnalise le contrôle social en créant une distance plus grande que jamais entre les agents et les commettants. Les centres de responsabilité sont plus diffus, plus flous, et se déplacent continuellement. 
Ce faisant, "l'équilibre du pouvoir dans le réseau des relations où s'insèrent les agences favorise les agents " (Shapiro, 1987 : 201). Moins les commettants ont de pouvoir pour mettre en application les ententes, plus ils doivent miser sur la confiance, plus ils sont susceptibles de devenir des « victimes involontaires d'abus de confiance » (Shapiro, $1987: 201$ ), et plus ils doivent s'en remettre aux institutions publiques pour demander en leur nom que l'équilibre soit restauré. Pour la tierce partie, le respect des droits s'avère encore plus difficile à obtenir. South (1983: 54-55) décrit bien le problème de la responsabilité des services de sécurité privée :

La nature commerciale et instrumentale du secteur de la sécurité privée... le rend particulièrement sujet à opérer selon un ethos, qui se justifie de lui-même, de la nécessité de recourir aux raccourcis, de fournir des services non conventionnels pour lesquels il y a une demande et d'éviter la procédure et les formes légales rigoureuses... L'industrie de la sécurité privée remplit un espace structurel entre les services de police de l'État et la surveillance que l'on reprend à son propre compte... Cet espace est investi par des entrepreneurs commerciaux qui partagent une idéologie conservatrice, une vision pragmatique du secteur du service, axée sur la préservation de l'ordre public, motivée et corrompue par l'appât du gain.

Shapiro (1987: 204) fait remarquer avec ironie que ce sont les limites mêmes des personnes cherchant à se protéger et à protéger leurs biens qui les incitent à engager des services de sécurité qui, simultanément, restreignent leur pouvoir d'exiger le respect des conditions du contrat. Shapiro soutient que les modèles de contrôle traditionnels du respect des clauses contractuelles, par la dissuasion ou par voie de restitution via les procédures civiles, sont presque totalement inadéquats pour lutter contre les abus de confiance dans le secteur des services de sécurité. Les stratégies de contrôle social traditionnelles, les cadres légaux et institutionnels courants sont, au mieux, trop lents, attendant l'échec flagrant des responsables d'honorer leurs engagements futurs, et, au pire, ignorent complètement les méfaits plus subtils et complexes. Dans les causes de fraudes et d'inconduites décrites plus haut, la malhonnêteté était généralement révélée par hasard, par des informateurs ou des journalistes d'enquête, ou de façon ponctuelle, dans le cadre d'une enquête spéciale. Les fraudes et les abus de pouvoir ne font pas l'objet de procédures routinières de réglementation interne ou externe et demeurent souvent inconnus pendant de nombreuses années. Les recours en poursuite civile ou criminelle, de leur côté, produisent des résultats très limités qui arrivent trop tard pour réparer tous les dommages.

En Australie, les entreprises de sécurité privée sont assujetties aux pratiques et aux lois sur les corporations de l'État et de la fédération et aux dispositions du droit criminel touchant la protection des biens et de la 
personne. Les agents qui portent des armes sont régis par la législation de l'État en cette matière. Le marché impose aussi un mécanisme forçant l'industrie à répondre de ses actes. Ces mesures de contrôle sont plus étendues qu'elles ne le paraissent, bien qu'elles ne soient pas adéquates, étant trop dépendantes des connaissances des clients et des plaintes rétrospectives (Reynolds et Wilson, 1996). Les problèmes allégués dans l'industrie proviennent en partie du fait que la sécurité est un domaine où les prestataires de services peuvent établir leur commerce sans posséder, ou presque, les qualifications nécessaires, et où les consommateurs n'ont pas les moyens de vérifier leurs références (South, 1983, 1988). Pour reprendre une citation de la police de la Nouvelle Galles du Sud : les personnes et les compagnies sont « présentement capables d'entrer dans l'industrie de la sécurité... sans aucune expérience ni connaissance technique, sans aucun sens des affaires, sans qualifications, sans engagement et sans intégrité, ou si peu » (NSWPS, 1995 : $10)$.

L'autoréglementation a traditionnellement été le mécanisme de réglementation principal opérant au-dessus du cadre légal courant en matière de sécurité privée. Bien que les organismes régissant l'industrie soient préoccupés par leur image de marque et s'engagent en principe à respecter des normes strictes de conduite, ils tendent à adopter une attitude passive à l'égard de la surveillance des agissements de leurs membres. Les organismes de régie ne peuvent faire beaucoup plus que de retirer leur carte d'adhésion aux membres fautifs, mesure à laquelle ils ont rarement recours (South, 1988). Lors d'une étude récente sur l'industrie de la sécurité australienne, on a découvert que $53 \%$ des compagnies ne sont pas affiliées à une association et que $14 \%$ seulement sont membres d'un organisme national. Près de $70 \%$ des petites entreprises ne sont en aucune façon affiliées à une association (Prenzler, 1995). Le rapport de l'ICAC sur la divulgation illégale de renseignements confidentiels a sévèrement critiqué l'Institute of Mercantile Agents et la National Association of Investigators, le président de cette dernière ayant été impliqué dans une affaire illicite, comme l'avaient été des membres et des agents anciens et actuels de l'Institute of Mercantile Agents. Le rapport affirme que «l'industrie ne peut être considérée comme étant apte à réglementer ses propres affaires et à établir et maintenir des normes de conduite satisfaisantes » (ICAC, $1992: 128)$.

On a, à l'occasion, proposé la déréglementation pour améliorer la qualité du service par la pression du marché et la réduction de l'autorité gouvernementale, mais ce projet n'a pas reçu grand appui. L'exemple le plus récent en Australie est le « rapport Bartley » (Deregulation Unit, 1990) qui recommande l'abandon du système actuel d'octroi des permis des agents commerciaux, des enquêteurs privés et des huissiers. De son côté, le rapport de la 
Independent Commission Against Corruption prétend que le rapport Bartley est extrêmement naîf et recommande un «contrôle plus serré » de la part des organismes gouvernementaux de régie des permis (ICAC, 1992 :10, 124128). Le rapport de la police de la Nouvelle Galles du Sud conclut également que « le moment n'est pas encore venu d'accorder à l'industrie des responsabilités d'autoréglementation importantes » (NSWPS, 1995) et cite à l'appui une enquête de 1983 sur la sécurité privée dans l'État de NewYork qui soutient que «peu d'emplois offrent des perspectives aussi prometteuses pour les délinquants potentiels ». Le droit civil, le droit des corporations et le droit criminel, de même que l'autoréglementation et la déréglementation, sont apparus inadéquats et auraient même contribué à faciliter les conduites illicites en créant une illusion de protection.

\section{La réglementation des relations de confiance}

Les problèmes dans l'industrie de la sécurité concernent avant tout la question de la responsabilité qui, à son tour, mène à la question de la réglementation spéciale (South, 1988). Comme dans la plupart des pays qui ont une administration fédérale, la réglementation australienne est hautement incohérente d'un État à l'autre. La formation obligatoire en toute matière se limite à une période d'une semaine ou moins. Dans la plupart des États, la réglementation exclut de grands secteurs de l'industrie, comme les serruriers, les installateurs de système d'alarme, le personnel des postes de surveillance et les agents de sécurité internes. Les ressources des agences chargées de l'application de la loi ne sont pas suffisantes et ces dernières n'ont pas pris l'initiative de vérifier le respect des clauses contractuelles, pas plus qu'elles n'ont poursuivi la professionnalisation des secteurs réglementés - une situation qui s'éloigne de l'opinion de l'ICAC voulant que les «fonctionnaires (de l'agence de réglementation) contribuent largement à l'efficacité, aux normes et à la réputation de l'industrie » (1992: 132).

Certains cas décrits plus haut ont donné lieu à des révisions législatives dans les États respectifs où ils se sont produits, qui ont institué l'adoption de permis élargis, la formation obligatoire et la vérification des dossiers criminels. Néanmoins, la réglementation en Australie demeure partielle, incohérente et largement passive. Aussi, devant les insuffisances du processus d'application de la réglementation spéciale existante, on demande que le gouvernement joue un plus grand rôle et exerce un contrôle plus strict sur l'industrie afin de mieux protéger les clients. Globalement, on semble favoriser le modèle de régime de permis élargi à l'échelle nationale et internationale (South, 1988 ; Fisher et Green, 1992 ; Wilson, 1994 ; George et Button, 1997). La demande de réglementation élargie est fondée sur l'importance de la sécurité pour la protection et la liberté des personnes, le 
besoin d'élaborer de hautes normes en matière de conduite et de service à la clientèle et le danger des abus.

Dans quelle mesure la réglementation nuit-elle à la productivité et occasionne-t-elle une opposition intrinsèque entre ceux qui réglementent l'industrie et ceux qui sont visés par la réglementation? Grabosky et Braithwaite (1986) décrivent diverses méthodes de réglementation inefficaces, y compris le detached token enforcement et la réglementation imposée. La réglementation pourrait échouer en raison de l'aliénation de l'industrie et de l'émergence de pratiques subversives, comme les tentatives de contrôler les organismes de réglementation ou de les intimider. Dans la plupart des pays occidentaux, la réglementation souffre souvent du paradoxe de la coexistence de pouvoirs de réglementation immenses et de pouvoirs d'exécution très limités, ceci souvent en raison de la déférence envers les prérogatives commerciales et la dépendance sociale à l'égard du commerce et de l'industrie qui assurent emplois et prospérité. Ainsi, Ayres et Braithwaite (1995) estiment que les formes de réglementation ouverte sont les plus susceptibles d'assurer la conformité, sans antagonisme. Cela signifie que l'industrie devrait intérioriser les normes et participer à leur élaboration.

On pourrait envisager une production conjointe de la réglementation pour favoriser un certain autocontrôle, selon Ayers et Braithwaite (1995). Cependant, le gouvernement a la responsabilité de protéger les droits des consommateurs et ne doit pas adopter de lois servant les intérêts de l'industrie de la sécurité ou d'un de ses secteurs. Le rapport de l'ICAC (1992 : 133) affirme : «Comme pour toutes les industries, on vise la participation maximale de l'industrie et des groupes de consommateurs. Mais cette industrie (agents d'enquête) a actuellement besoin, en raison de sa nature et de son histoire récente, d'un contrôle externe ». Néanmoins, l'expérience australienne confirme l'opinion que l'industrie de la sécurité, en particulier les associations professionnelles, désire réellement collaborer à l'amélioration de la réglementation (NSWPS, 1995 ; Prenzler et Hayes, 1998). L'appui des plus petites entreprises dément l'argumentit voulanl que le lobbying des permis constitue une stratégie anticoncurrentielle des entreprises de plus grande taille (South, 1983). Le morcellement des associations de l'industrie et l'incapacité d'établir une organisation unifiée ou des principes directeurs constituent un obstacle majeur aux efforts de réglementation conjointe. Mais les données confirment que le gouvernement peut $s$ 'attendre à des propositions pratiques de réglementation honnêtes et généralement responsables de la part de l'industrie, axées sur l'intégrité et la compétence des agents et agences dans le respect de l'intérêt des consommateurs.

Dans un sondage récent mené en Australie, $96 \%$ des directeurs de la sécurité se disaient d'avis que le législateur devrait édicter des normes 
minimales de formation pour les agents armés, alors que $88 \%$ étaient d'accord avec cette proposition en ce qui concerne les agents non armés. C'est un très fort appui (vision commune dans tous les secteurs de l'industrie), compte tenu de l'hostilité du secteur privé à l'égard de l'intervention gouvernementale. Un grand nombre de directeurs (42\%) seraient en faveur d'un certificat collégial technique d'un an, ce qui surpasse de loin les exigences actuelles du marché du travail en sécurité (Prenzler, 1995). Le sondage a également mis à jour un accord très marqué pour la formation obligatoire des cadres des services de sécurité - un secteur de travail où l'on doit s'attendre à ce que l'idéologie de contrôle du marché prédomine. Les répondants ont accepté, dans une proportion de $88 \%$, la proposition d'adoption de normes minimales de formation des cadres. Les chercheurs s'interrogent sur l'application possible des exigences de formation en ce qui les concerne, mais les témoignages en faveur de cette proposition sont de plus en plus nombreux. Le rapport du VCCAV (1990) a fait ressortir que les cadres faisaient preuve de négligence en permettant des manifestations de violence dans les débits de boisson et en acceptant qu'une partie de cette violence soit le fait du personnel de sécurité employé pour la prévenir. Enfin, le rapport de l'ICAC (1992) notait, pour sa part, que les cadres des secteurs public et privé devraient prendre davantage conscience de leur obligation de prévenir les inconduites et recommandait de resserrer la définition de la responsabilité légale des cadres à l'égard des interventions de leurs employés. Le rapport de la Police de la Nouvelle Galles du Sud (1995 : ii) recommandait l'adoption d'un « contrat de fidélité garantissant les connaissances appropriées, les ressources financières et le sérieux des personnes qui présentent une demande de permis pour cette catégorie " (individus et agences). Des codes de conduite très spécifiques et exécutoires pourraient aussi permettre de prévenir les abus dont sont victimes les consommateurs de services de sécurité, comme dans le cas du meurtre décrit plus haut.

Les définitions de comportements sans scrupule pourraient inclure l'omission, de la part des fournisseurs de services de sécurité, de s'informer de l'usage légal de leurs produits de sécurité. La vérification des dossiers criminels est au cour de l'adoption de la nouvelle législation. Cependant, il faut aborder cette proposition avec scepticisme du point de vue de la dissuasion et de la stigmatisation, car peu de contrevenants sont reconnus coupables, et ce pour la plupart des catégories d'infractions. Les prédictions de récidive sont très imprécises et les contrevenants devraient avoir droit à la réintégration plutôt qu'à la stigmatisation et à l'exclusion. Néanmoins, toutes les parties concernées semblent convenir que la vérification des dossiers criminels (pour les 10 dernières années environ) est un élément essentiel de la réglementation (NSWPS, 1995 ; Prenzler et al., 1997 ; Prenzler et al., en préparation). 
L'incidence des changements apportés à la législation en Australie n'a pas fait l'objet de contrôles préliminaires ou ultérieurs adéquats. Tout au plus, le corps de police de Victoria chargé de l'administration du Private Agents (Amendment) Act de 1990, adopté à la suite de l'enquête sur la violence de 1990 , s'est contenté de faire une remarque sibylline, disant que « l'effet à court terme a été considérable » (Frost, 1991 : 16). Une étude sur les gérants des boîtes de nuit du Queensland conclut que le Security Providers Act de 1993 a incité le personnel de la sécurité à adopter une meilleure conduite (Prenzler et Hayes, 1998). Reste qu'actuellement, il est purement spéculatif et anecdotique de prétendre à l'efficacité d'un meilleur contrôle, tâche qui représente un défi pour les évaluateurs. Néanmoins, on discute d'un nouveau projet de législation concernant la sécurité privée qui jouit d'un grand appui et dont voici les éléments essentiels :

- la législation toucherait l'ensemble de l'industrie - tous les secteurs de la sécurité autres que ceux qui ont leur propre forme de spécialisation et de contrôle, comme la police et les militaires. Il reste à savoir s'il faut englober les autres catégories d'emplois liés à la sécurité, comme le personnel administratif des entreprises de sécurité, mais tous les intervenants principaux devraient être visés, y compris les installateurs de systèmes d'alarme, les opérateurs de postes de contrôle, les consultants et les agents sur place ;

- la législation serait de portée nationale et exigerait que les États et les territoires approuvent des lois et des règlements similaires, ce qui autoriserait le transfert des permis d'un État à l'autre et empêcherait les personnes à qui on a refusé l'octroi d'un permis dans une autre unité administrative de contourner la loi ;

- la législation permettrait l'exclusion des personnes qui ont un casier judiciaire, ou qui n'ont pas respecté les conditions d'utilisation du permis ;

- la législation fixerait des normes de formation obligatoires fondées sur une analyse des tâches des agents de sécurité et des qualifications de base. II faudrait toutefois reconnaître les qualifications et la formation antérieure des agents actuels ;

- la législation imposerait l'obligation de rendre des comptes, par des mesures de surveillance des enquêtes sur les plaintes, un code de conduite et même la surveillance du paiement du salaire minimum;

- la législation prévoirait des mesures de redressement des torts causés. Les ressources financières pour les compensations aux victimes proviendraient d'une caution financière versée par le contrevenant.

Le modèle serait de type consultatif et établirait un comité permanent composé des représentants de l'industrie et des principaux concernés qui 
conseilleraient l'agence de réglementation. L'agence de réglementation aurait pour mandat de mener des recherches et de promouvoir la professionnalisation, ce qui entraînerait une qualification plus grande du secteur de la sécurité.

\section{Services de sécurité permanents ou contractuels ?}

Une autre question concernant le problème de la confiance dans l'industrie de la sécurité touche les mérites relatifs des services de sécurité permanents par rapport aux services de sécurité contractuels. Il est urgent que le gouvernement se penche sur cette question, compte tenu de la tendance du secteur public australien de réduire les services de sécurité permanents et de confier cette tâche à l'entreprise privée. Étonnamment, peu de recherches empiriques ont été menées sur ce sujet crucial.

Un des attraits de la sécurité privée est son coût nettement moins élevé, qui s'explique en partie par de plus grandes économies d'échelle en matière de recrutement, d'administration, d'assurance et de formation par rapport à la capacité de payer de nombreux clients. Les services de sécurité privée sont également intéressants pour combler des besoins à court terme, pour remplir des fonctions spéciales ou encore en réponse à des situations de crise. Il est plus facile d'en réduire les effectifs lorsque cela est nécessaire. Le roulement de personnel peut aider à prévenir la construction d'empires, les conseils partiaux ou la fraude des agents permanents.

Les critiques ripostent en disant que les services de sécurité privée offrent des économies à court terme qui pourraient ne plus en être à long terme : les coûts de main d'œuvre plus bas pourraient résulter en une formation réduite, de moins bonnes conditions de travail, et des salaires inférieurs au salaire minimum ; il serait plus difficile d'évaluer la qualité des services rendus surveillance des relations de confiance - en outre la surveillance du respect des clauses contractuelles pourrait induire une administration cachée, étant donné que la délégation de la responsabilité est un peu plus loin de la source. En outre, on suggère que le personnel de sécurité permanent est peut-être plus qualifié et compétent, compte tenu de la plus grande sécurité d'emploi et des meilleures conditions de travail que procure un emploi permanent, et du fait que l'on peut procéder à une meilleure sélection du personnel et le former pour les besoins particuliers de l'organisation.

Les récits anecdotiques que nous avons recueillis indiquent que les agents permanents sont plus loyaux et ont une connaissance précieuse des lieux où ils travaillent. En outre, le travail des agents de sécurité permanents peut avoir une plus grande portée et englober la santé et la sécurité sur les lieux du travail, la prévention des accidents et des incendies, et la détection des besoins 
de maintenance. Les services permanents de sécurité présentent peut-être aussi une meilleure image aux clients de l'organisation, plutôt que celle de renta-cop ou de tueur à gages (Fisher et Green, 1992 ; Kennedy, 1995).

Les arguments en faveur de services d'agents de sécurité permanents s'appuient sur les résultats d'une étude de Button et George (1994) réalisée auprès des organismes qui emploient des agents permanents, parmi lesquels plusieurs auraient vécu une expérience insatisfaisante avec des gardes de sécurité privée en raison du changement fréquent de personnel, du manque de formation et de motivation, et de la connaissance insuffisante des lieux surveillés. De nombreuses initiatives réussies de prévention de la criminalité, bien documentées, sont le fait d'agents de sécurité permanents qui ont pris part aux recherches et expériences sur les lieux pour examiner tous les éléments de perte potentielle et proposer des mesures adaptées afin de les prévenir (Clarke, 1992).

Une étude sur l'observation du contrôle des foules lors de grands événements sportifs, à Melbourne, suggère également que les agents de sécurité permanents maintiennent mieux l'ordre. Il semble que l'emploi d'agents permanents incite les directeurs du stade à participer directement à la planification de la sécurité et à la formation de leurs préposés à la sécurité. Comparant deux comportements de foule lors de deux matches de football, Warren (sous presse) commente :

Le succès des pratiques de sécurité aux parties de la ligue nationale de football est peut-être attribuable à l'emploi d'agents permanents, qui encourage une plus grande vigilance des directeurs et des lignes plus directes de responsabilité entre les organisateurs des événements et les contrôleurs de foule privés qu'il n'est possible dans le modèle de services contractuels adopté par la Ligue de football de l'Australie.

L'étude de la violence dans les débits de boisson de Victoria a d'ailleurs révélé que le recours aux services de sécurité privée sur une base contractuelle a tendance à aggraver la mauvaise gestion du maintien de l'ordre. Dans certains cas, les détenteurs de permis qui avaient engagé des agents de sécurité ne connaissaient même pas le nom des contrôleurs de foule et semblaient ne prendre aucune responsabilité quant à la conduite du personnel de la sécurité (VCCAV, $1990: 48$ ).

En somme, il semble que les services d'agents de sécurité permanents conviennent particulièrement aux organisations dont les besoins de sécurité sont relativement stables et qui désirent aborder les problèmes de sécurité de façon systématique, voire scientifique. Par ailleurs, l'avantage majeur des contrats de sécurité est la souplesse qu'ils offrent aux usagers. Dans ces conditions, il n'est pas surprenant que le Hallcrest Report constate que 
certaines entreprises offrent une forme hybride de services de sécurité avec des services de base permanents et la gestion, par l'organisation, des agents contractuels (Cunningham et al., 1990 ; Button et George, 1994).

\section{CONCLUSION}

L'étude des cas récents de pratiques de fraude, d'inconduite et de raccourcis dans l'industrie de la sêcurité privée en Australie illustre de façon inquiétante l'ampleur des possibilités d'abus de confiance dans les domaines où la protection et la sécurité sont des enjeux importants. L'intervention accrue du gouvernement devrait redonner un plus grand contrôle sur les contrats de services de sécurité aux consommateurs qui sont seconde ou tierce parties. La réglementation n'est pas infaillible et les clients demeureront extrêmement vulnérables aux abus de confiance. Néanmoins, il existe des modèles de réglementation prometteurs qui indiquent clairement aux prestataires des services de sécurité que la société s'attend à des normes de probité extrêmement élevées. Dans cette perspective, l'industrie de la sécurité jouira d'une moins grande liberté, fera l'objet d'une surveillance étatique accrue et de normes plus strictes de qualification des personnes qui désirent travailler dans ce secteur. La réglementation du marché sera renforcée par une intervention accrue des États. Les contrôles exercés sur les candidats limiteront la compétition mais forceront une concurrence plus franche sur un pied d'égalité et excluront ceux qui refusent d'adhérer aux règles de conduite. Il en résultera une industrie plus professionnelle qui jouira d'une meilleure image de compétence et d'intégrité, d'une plus grande confiance du public et qui sera obligée de rendre des comptes.

\section{REFÉRENCES}

ABS (1988), Security/Protection and Other Business Services Industries, Mai, Canberra, Australie, Bureau of Statistics.

ABS (1996), Labour force estimates, Canberra, Australian Bureau of Statistics.

ABS (1997), Special Data Service, January, Canberra, Australian Bureau of Statistics.

ACCC (Australian Competition and Consumer Commission) (1996a), ACCC Journal, vol. 1, p. 42.

ACCC (1996b), ACCC Journal, vol. 4, p. 21-22.

ACCC (1996c), ACCC Journal, vol. 5, p. 26-27.

ACT (1992), Discussion paper : Issues and Policy Options in the Regulation of the ACT Security (Protection) Industry, Canberra, Attorney General's Department, Australian capital territory.

AYERS, I. et BRAITHWAITE, J. (1995), Responsive Regulation: Trancending the Deregulation Debate, New York, Oxford University Press. 
BUTTON, M. et GEORGE, B. (1994), «Why some organisations prefer in-house to contract security staff $\gg$ in GILL, M. (dir.), Crime at Work, (p. 210-223), Leicester, Perpetuity.

CLARKE, R. (1992), Situational Crime Prevention: Successful Case Studies, New York, Harrow and Heston.

CUNNINGHAM, W. S, STRAUCHS, J. et van METER, C. (1990), Private Security Trends 1970 to 2000, Boston, Butterworths-Heinemann.

DEREGULATION UNIT (1990), Report on Licensing Aspects of the Security (Protection) Industry Act, 1985, Sydney, NSW Goverrment.

FAY, J. (1993), «Budgeting : an overview » in FAY J. (dir.) Encyclopedia of Security Management, Stoneham, Butterworth-Heinemann.

FISHER, R. et GREEN, G. (1992), Introduction to Security, Boston, ButterworthHeinemann.

FROST, S. (1991), «Regulation of the private security industry and expectations of training authorities on security training ", Communication présentée à la Third South Pacific Region Security Conference and Exhibition, 16 au 18 juillet.

GEORGE, B. et BUTTON, M. (1997), « Private security industry regulation : lessons from abroad for the United Kingdom ». International Journal of Risk. Security and Crime Prevention, vol. 2, n' 3, p. 187-200.

GOLSBY, M. (1998), « Formalising co-operation in crime prevention », Security Journal, vol. $9, \mathrm{n}^{\circ} 1$, sous presse.

GRABOSKY, P. et BRAITHWAITE, J. (1986), Of manners Gentle : Enforcement Strategies of Australian Business Regulatory Agencies, Melbourne, Oxford University Press.

ICAC (1992), Report on the Unauthorised Release of Information, vol. 1, Sydney, Independent commission against corruption.

IRCNSW (1996), Matter No. IRCI880 of 1995, Reference by the Minister for Industrial relations pursuant to SD.345(4) of the Industrial Relations Act 1991 regarding the transport and delivery of cash and other valuables industry, Sydney, Industrial relations commission of NSW.

JOHNSTON, L. (1992), The Rebirth of Private Policing, London, Routledge.

KENNEDY, D. (1995), «A synopsis of private security in the United States », Security Joumal, vol. 6, p. 101-105.

LUMLEY GENERAL INSURANCE LTD, M. MARSH et A.K. BUSHNELL VS G. ROSENBERG et ORS, Brisbane Magistrates Court, 20-21 octobre 1994.

MORLEY, T. (1994), « Private security or privatte police? » in BILES D. et VERNON, J. (dir.) Private Sector and Community Involvement in the Criminal Justice System, Canberra, Australian institute of Criminology.

NSWPS (1995), Security industry review, Sydney, NSW Police Service.

POLK, K. et RANSON, D. (1991), "The role of gender in intimate homicide ", Australian and New Zealand Journal of Criminology, vol. 24, $\mathrm{n}^{\mathrm{s}} 1$, p. 15-24.

POST, R. et KINGSBURY, A. (1991), Security Administration, Boston, ButterworthHeinemann.

PRENZLER, T. (1995), "Security manager's perceptions of industry regulation : an Australian study, Security journal, vol. 6, $\mathrm{n}^{\mathrm{D}} 2$, p. 227-234.

PRENZLER, T., BAXTER, T. et DRAPER, R. (1997), « Special legislation for the security industry : a case study ", International Journal of Risk, Security and Crime Prevention, vol. $2, \mathrm{n}^{\circ} 3$, p. $19-31$. 
PRENZLER, T. et HAYES, H. (1998), « Nightclub managers and the reguiation of crowd controllers », Security Joumal, vol. $9, \mathrm{n}^{\circ} 1$.

PRENZLER, T., HAYES, H. et WORTLEY, R. (en préparation), Evaluation of the Queensland Security Providers Act : Report to the Criminology Research Council.

PRIVATE COPS (1994), Lateline, ABC TV, 12 Juillet.

PRIVATE COPS (1996), Report of the Queensland Industrial Relations Commission : Industrial Matters Relating to the Chase-in-Transit Industry in Queensland, Brisbane, Division of Workplace, Health and Safety, WIRC.

QIRC (1996), Report of the Queensland Industrial Relations Commission : Industrial Matters Relating to the Cash-In-Transit Industry in Queensland, Brisbane, Division of Workplace Health and Safety, QIRC.

REES, R. (1984), Private Security in Australia, Canberra, Australian institute of criminology. RENT-A-COP (1995), Background Briefing, 16 juillet, Sydney, ABC Radio National.

REYNOLDS, C. et WILSON, P. (1996), "Private policing : creating new options ", in CHAPPELL, D., et WILSON, P. (dir.). Australian Policing : Contemporary Issues, Sydney, Butterworths.

SARRE, R. (1994), " The Legal Basis for the Authority of Private Police and An Examination of Their Relationship with the 'Public' Police », in BILES, D. et VERNON, J. (dir.), Private Sector and Community Involvement in the Criminal Justice System, Canberra, Australian Institute of Criminology.

SARRE, R. (1998), «Accountability and the private sector : putting accountability of private security under the spotlight ». Security Journal, vol. $9, \mathrm{n}^{\mathrm{n}} \mathrm{I}$ (sous presse).

SHAPIRO, S. (1987), « Policing trust » in SHEARING, C. et STENNING, R. (dir.), Private Policing, Newbury Park, California, Sage.

SHEARING, C. et STENNING, P. (1983), Private Security and Private Justice : the Challenge of the 80 's, Montreal, Institute for research on public policy.

SOUTH, N. (1983), "The comuption of commercial justice : the case of the private security sector », in CLARKE, M. (dir.) Corruption : Causes, Consequences and Control, New York, St-Martin's.

SOUTH, N. (1988), Policing for Profit, London, Sage.

STENNING, P. (1994), « Private policing : some recent myths, developments and trends », in BILES D. et VERNON, J. (dir.), Private Sector and Community Involvement in the Criminal Justice System, Canberra, Australian institute of Criminology.

SWANTON, B. (1993), «Police and private security : possible directions », Trends and Issues in Criminal Justice, Février, Canberra, Australian institute of criminology.

TPC (1995), Trade practices commission bulletin, vol. 82, p. 35-36, Canberra, TPC.

VCCAV (1990), Inquiry Into Violence In and Around Licensed Premises, Melbourne, Victorian community council against violence.

WAP (1997), Partnership in Policing : Alarm Response Working Group Policy, Perth, Western Australia Police.

WARREN, I. (1998), «Patron cultures, policing and security : Trends from two Australian sport sites », Security Joumal, vol. 9, no. 1.

WILSON, P. (1994), «The australian private security industry : the need for accountability, regulation and professionalism " in BILES D. et VERNON J. (dir.), Private Sector and Community Involvement in the Criminal Justice System, Canberra, Australian institute of criminology. 